\title{
QALYs and HYEs: Under what conditions are they equivalent?
}

\author{
Han Bleichrodt *
}

Institute for Medical Technology Assessment, Department of Health Policy and Management, Erasmus University, P.O. Box 1738, 3000 DR Rotterdam, The Netherlands

Received July 1993; revised December 1993

\begin{abstract}
The paper examines what restrictions have to be imposed on the individual's preference structure for QALYs and HYEs to yield identical results. It is shown that using QALYs involves imposing three additional restrictions. Empirical evidence suggests that these restrictions cannot be expected to hold in all applications. The main problem in using HYEs appears to be practical. An alternative index is proposed, that may help to bridge the gap between QALYs and HYEs by combining to some extent the advantages of the two measures.
\end{abstract}

Keywords: Utility theory; Quality adjusted life years; Healthy years equivalents; Cost-utility analysis JEL classification: $\mathbf{1 1 0}$

\section{Introduction}

The evaluation of health care programs involves both technical and value judgements. The value judgements concern mainly the trade-off between the two important outcomes of such programs: quality of life gained and quantity of life gained.

\footnotetext{
*Tel: + 3110 4088555, Fax: +31 10 4522511, E-mail: bleichrodt@econ.bmg.eur.nl
} 
The QALY approach offers one way of incorporating these two benefits of health care programs into one single index measure: quality adjusted life years gained. On the basis of this index decisions concerning the allocation of resources in the health care sector can be made. The program that should be implemented is the one that offers the largest number of QALYs per dollar or, what is equivalent, the one that has lowest costs per QALY gained.

The QALY approach has been criticized by several authors (e.g. Loomes and McKenzie, 1989; Mehrez and Gafni, 1989). The essence of this criticism is that since QALYs rely on certain fairly restrictive assumptions (Pliskin et al., 1980; Weinstein et al., 1980) as a representation of individuals' preferences, care should be taken in using them in the evaluation of health care programs. Mehrez and Gafni (1989) propose an alternative index, the Healthy Years Equivalents (HYEs), which as they claim, fully represents patients' preferences, stemming from the way they are calculated from each individual's utility function. At the same time HYEs retain some attractive properties of QALYs: combining quality of life and quantity of life in a single index and being easy to interpret.

The aim of this paper is to show how QALYs and HYEs are related to each other, that is, under what assumptions about the underlying preference structure they will give identical results. Both the certainty case and the uncertainty case will be considered. Moreover, it will be argued that the claim that HYEs fully represent an individual's preferences is not completely true. Even HYEs make simplifying assumptions concerning the individual's preference structure. Besides this theoretical reservation, the main problem in implementing HYEs to evaluate health care programs appears to be practical. An alternative index is proposed, which may help to bridge the gap between QALYs and HYEs by combining to some extent the advantages of the two measures.

\section{Quality-adjusted life years}

The basic QALY model, as it is typically encountered in the literature, is simple. Abstracting from discounting, ${ }^{1}$ denote by $q_{t}$ the health status level in period $t$, where it is assumed without loss of generality that each period lasts one year. Assume that health status levels form a continuum. The number of QALYs

\footnotetext{
${ }^{1}$ Two things should be noted. First, discounting can be accounted for within this framework by imposing an additional stationarity assumption (Koopmans, 1972). Second, it is not clear that discounting of QALYs is necessary. Following the suggestions made by Torrance and Feeny (1989), health state utilities are measured over the individual's lifetime. Measuring health state utilities this way clearly incorporates time preference.
} 
represented by the lifetime health stream $Q_{T}=\left(q_{1}, \ldots, q_{t}, \ldots, q_{T}\right)$ where $T$ is the number of years to live from now on, is

$$
\mathrm{QALY}=\sum_{t=1}^{T} u\left(q_{t}\right),
$$

where $u\left(q_{t}\right)$ is the utility associated with health status level $q$ in period $t .^{2}$

By computing (1) for the various programs and dividing this amount by the costs one arrives at the decision rule maximize QALYs per unit of costs.

In the rest of this paper I will refer to Eq. (1) as the QALY model. I will derive under what conditions an individual's preferences can be represented by this utility function. One could object against this that by taking the basic version of the QALY model as the QALY model, it is a bit like assuming that the micro-economics of the firm is locked into the perfect competition assumptions. Meeting this objection requires an assessment of which assumptions are essential for the QALY concept and which are made for convenience (ease of measurement). I will briefly comment on this in Section 7 .

\section{Healthy years equivalents}

The calculation of HYEs is slightly more involved. Denote the lifetime health stream again by $Q_{T}=\left(q_{1}, \ldots, q_{t}, \ldots, q_{T}\right)$. The utility function over this stream is $U\left(Q_{T}\right)$, which represents the utility as viewed now by the individual. Denote $q^{*}$ as the best health state (generally perfect health) and $q^{0}$ as the worst health state (generally death ${ }^{3}$ ). Let $H$ be the number of years in $q^{*}$ and $H^{*}$ be the healthy-years equivalent of $Q_{T}$. The problem is now to find $H^{*}$ such that

$$
U\left(Q_{H^{*}}\right)=U\left(Q_{T}\right)
$$

where $Q_{H}$ * is a lifetime health profile with $q_{t}=q^{*}$ for $t=1, \ldots, H^{*}$ and $q_{t}=q^{0}$ for $t=H^{*}+1, \ldots, T$.

In case the vNM axioms hold, Mehrez and Gafni (1991) have shown that HYEs can be measured by a two-stage lottery-based procedure.

\footnotetext{
${ }^{2}$ It has been pointed out to me by one of the referees that this equation is not correct, given that in health care evaluation it is the gain in QALYs that has to be measured rather than the total number of QALYs. However, this does not affect the results of this paper. Differences between utilities are only meaningful if additional axioms on top of the conditions derived in this paper are imposed on the individual's preference structure (see for example Krantz et al., 1971).

${ }^{3}$ Death need not necessarily be the worst health state. However, health states worse than death cause major theoretical problems. In the context of the QALY model health states worse than death cause a violation of one of the assumptions underlying the QALY model: mutual preferential/utility independence (see below). In the HYE model such health states may lead to negative values for the number of HYEs associated with a health care program.
} 


\section{The certainty case: Value functions}

What conditions have to be imposed on the individual's preference structure to make QALYs and HYEs equivalent? First consider the case where the outcomes of a medical intervention are certain. In the case of certainty, an individual's preferences can ideally be captured by a value function which has the following properties:

$$
\text { if } \boldsymbol{x} \geqslant \boldsymbol{y} \text { then } v(\boldsymbol{x}) \geq v(\boldsymbol{y}),
$$

and

$$
\text { if } \boldsymbol{x} \succ \boldsymbol{y} \text { then } v(\boldsymbol{x})>v(\boldsymbol{y}),
$$

where $\boldsymbol{x}$ and $\boldsymbol{y}$ are vectors of attributes from which the individual derives value, one of which is health. If the individual's behaviour satisfies certain axioms (Debreu, 1954, Debreu, 1964) such a value function can be shown to exist.

The QALY approach, again abstracting from discounting, assumes the following value function to measure individual preferences for a lifetime health stream $Q_{T}$ :

$$
v\left(Q_{T}\right)=\sum_{t=1}^{T} v\left(q_{t}\right),
$$

which is an additively separable value function.

Before deriving the assumptions sufficient for such an additive form to be a correct representation of the individual's preferences under certainty, it is useful to introduce some terminology. Suppose that there are $n$ attributes from which an individual derives value: $X_{1}, \ldots, X_{n}$. These attributes map each act $a$ into a point $X(a)=\left[X_{1}(a), \ldots, X_{n}(a)\right]$ in the $n$-dimensional consequence space. Suppose further that the vector of attributes $\boldsymbol{x}$ can be subdivided into two subvectors $\boldsymbol{y}$ and $\boldsymbol{z}$ where

$$
y=\left(x_{1}, \ldots, x_{r}\right) \text { and } z=\left(x_{r+1}, \ldots, x_{n}\right) .
$$

Consider two values for the vector $y, y^{1}=\left(y_{1}^{1}, \ldots, y_{r}^{1}\right)$ and $\boldsymbol{y}^{2}=\left(y_{1}^{2}, \ldots, y_{r}^{2}\right)$, and one for the vector $z, z^{1}=\left(z_{r+1}^{1}, \ldots, z_{n}^{1}\right)$ then

Definition 1. $y^{1}$ is conditionally preferred or indifferent to $y^{2}$ given $z^{1}$ if and only if

$$
\left(y^{1}, z^{1}\right) \succcurlyeq\left(y^{2}, z^{1}\right) \text {. }
$$

Definition 2. The set of attributes $Y=\left\{X_{1}, \ldots, X_{r}\right\}$ is preferentially independent of the complementary set $Z=\left\{X_{r+1}, \ldots, X_{n}\right\}$ if and only if the conditional preference structure for $y$ given $z^{1}$ does not depend on $z^{1}$. Or, $Y$ is preferentially independent of $Z$ iff for some $z^{1}$

$$
\left[\left(y^{1}, z^{1}\right) \succcurlyeq\left(y^{2}, z^{1}\right)\right] \Rightarrow\left[\left(y^{1}, z\right) \succcurlyeq\left(y^{2}, z\right)\right], \forall z, y^{1}, y^{2} .
$$


Definition 3. The attributes $X_{1}, \ldots, X_{n}$ are mutually preferentially independent if every subset $Y$ of these attributes is preferentially independent of its complement.

Theorem 1 (Debreu, 1960). Iff the attributes $X_{1}, \ldots, X_{n}$ are mutually preferentially independent and $n \geq 3$, the value function is of the additive form

$$
v\left(x_{1}, \ldots, x_{n}\right)=\sum_{i=1}^{n} v_{i}\left(x_{i}\right)
$$

where $v_{i}$ is an additive value function over $X_{i}$.

Generally, $v$ and each of the single-attribute value functions $v_{i}$ are scaled from 0 to 1 . Following this scaling convention the following form of the value function results

$$
v(\boldsymbol{x})=\sum_{i=1}^{n} \tau_{i} v_{i}\left(\boldsymbol{x}_{i}\right),
$$

where the $\tau_{i}$ are scaling constants.

In the case of QALYs, the attributes of the value function are health status levels in different years. Thus, the value function, which is now a value function for health, consists of $T$ attributes, health status levels in the various years $\left(q_{1}, \ldots, q_{T}\right)$ over which single-period value functions $v_{t}$ are defined.

To arrive at the basic QALY formulation two more assumptions besides mutual preferential independence have to be imposed on the individual's value function: (1) Stable preferences over lifetime, i.e. all single-period value functions are identical.

(2) All scaling constants are equal; this implies that improvements in health are equally important across periods.

The number of HYEs in the certainty case is calculated by finding the value of $H^{*}$ for which

$$
v\left(Q_{T}\right)=v\left(Q_{H} \cdot\right),
$$

where $Q_{T}$ and $Q_{H}$. are defined as before.

Since HYEs do not impose any additional ${ }^{4}$ restrictions on the individual's value function for health, QALYs and HYEs will yield identical results under the three assumptions derived above.

Both the QALY approach and the HYE approach assume that the value function for health exists. That is, health and non-health attributes in the individual's overall value function are assumed to be mutually preferentially independent.

\footnotetext{
${ }^{4}$ The HYE model, to give sensible results, has to impose a monotonicity condition: individual preferences have to be increasing with respect to healthy years of life. Note that this assumption is also assumed by the QALY model.
} 
In other words, preferences for lifetime health streams can be considered without consideration of other, non-health, attributes that bear value to the individual. Without this additional assumption, neither QALYs nor HYEs will correctly represent the individual's preferences under certainty.

\section{The uncertainty case: Utility functions}

Assume that expected utility is the appropriate criterion to use in choosing among alternatives ${ }^{5}$. That is, an individual's preferences can be captured by a utility function which has the property that given two probability distributions $A$ and $B$ over the multi-attribute consequences, probability distribution $A$ is at least as desirable as probability distribution $B$ iff,

$$
E a[u(\tilde{x})] \geq E b[u(\tilde{x})] .
$$

where $E a$ and $E b$ are expectation operators taken with respect to distributions A and $B$ respectively.

The utility function which describes the individual's behaviour is unique up to a positive linear transformation. Note that in the degenerate case, where one of the consequences occurs with probability one, the utility function reduces to a value function. Thus, a utility function is by definition a value function, but a value function is not necessarily a utility function.

The QALY approach assumes that the utility of the lifetime health stream $Q_{T}$ can be assessed by the following utility function:

$$
u\left(Q_{T}\right)=\sum_{t=1}^{T} u\left(q_{t}\right)
$$

which is an additively separable utility function.

An alternative way of representing the utility of the lifetime health stream $Q_{T}$ is by considering the two-attribute utility function $u(T, q)$ where $q$ is a constant health status level representing the stream of health status levels in the $T$ years. $q$ can be obtained, recall that health status has been assumed to form a continuum, by solving the following equation:

$$
u(q, \ldots, q)=u\left(Q_{T}\right) \text {. }
$$

This is the approach followed by Pliskin et al. (1980) (PSW). PSW consider the QALY representation

$$
u(T, q)=T u_{Q}(q),
$$

\footnotetext{
${ }^{5}$ Recently, the use of expected utility as the appropriate decision criterion has been challenged. For an overview of some recent developments in modelling preferences under uncertainty see for example Karni and Schmeidler (1991).
} 
where $u_{Q}$ represents the utility function for quality of life, and impose the following conditions on the individual's preference structure:

(1) mutual utility independence between life years and health status (assuming the latter is constant across periods);

(2) constant proportional trade-off of life years for health status;

(3) risk neutrality with respect to life years.

The definitions of utility independence and mutual utility independence are generalizations of Definitions 2 and 3 to the case of preferences defined over lotteries over attributes rather than over the attributes themselves.

Definition 4. Attribute $Y$ is utility independent of $Z$ when conditional preferences for lotteries on $Y$ given $Z$ do not depend on the particular level of $Z$.

Definition 5. Attributes $Y$ and $Z$ are mutually utility independent if $Y$ is utility independent of $Z$ and $Z$ is utility independent of $Y$.

The assumption of a constant proportional trade-off of life years for health status implies that the proportion of remaining life years one is willing to give up for a given improvement in health status does not depend on the number of remaining life years. Risk neutrality over life years implies a linear utility function for life years.

Assume that the individual's utility function for health exists, that is health and non-health attributes in the individual's overall utility function are mutually utility independent. Then the three conditions are sufficient for the QALY model to be a correct representation of the individual's utility function for health.

Returning to Eq. (12), which is an alternative way of describing the utility function for a life time health stream, it can be asked under what conditions the intertemporal utility function reduces to this simple additive form. In answering this question, an alternative set of conditions is imposed on the utility function for health, which together have the same effect as the PSW conditions: a QALY can be considered a utility. ${ }^{6}$

Assume first that the attributes in the utility function for health (that is, health status levels in the various periods) be mutually utility independent. This assumption allows by applying Theorem 6.1 in Keeney and Raiffa (1976) to write the utility function for health either in the additive form

$$
u(q)=\sum_{t=1}^{T} k_{t} u_{t}\left(q_{t}\right) \text { if } \sum_{t=1}^{T} k_{t}=1
$$

\footnotetext{
${ }^{6}$ Note again that this is only true if health and non-health attributes in the individual's overall utility function are mutually utility independent.
} 
or in the multiplicative form

$$
1+k u(q)=\prod_{t=1}^{T}\left[1+k k_{t} u_{t}\left(q_{t}\right)\right] \quad \text { if } \quad \sum_{t=1}^{T} k_{t} \neq 1,
$$

where $k$ and the various $k_{t}$ are scaling constants and $u(q)$ and the various $u_{t}\left(q_{t}\right)$ are normalized such that $u\left(q^{*}\right)=u_{t}\left(q_{t}^{*}\right)=1$ and $u\left(q^{0}\right)=u_{t}\left(q_{t}^{0}\right)=0$.

In order to decide whether the additive or the multiplicative form is appropriate, the condition of additive independence has to be checked.

Definition 7. Attributes $q_{1}, \ldots, q_{T}$ are additive independent if preferences over lotteries on $q_{1}, \ldots, q_{T}$ depend only on their marginal probability distributions and not on their joint probability distribution.

Theorem 2. The additive utility function

$$
u(q)=\sum_{t=1}^{T} k_{t} u_{t}\left(q_{t}\right)
$$

is appropriate iff the attributes are additive independent, where

(i) $u$ and $u_{t}$ are normalized such that $u\left(q^{*}\right)=1 ; u\left(q^{0}\right)=0 ; u_{t}\left(q_{t}^{*}\right)=1$ and $u_{t}\left(q_{t}^{0}\right)=0$

(ii) $k_{t}=u\left(q_{1}^{0}, \ldots, q_{t-1}^{0}, q_{t}^{*}, q_{t+1}^{0}, \ldots, q_{T}^{0}\right)$.

A sketch of the proof has been given in the Appendix.

Additive independence is a stronger restriction than mutual preferential independence. Additive independence implies mutual preferential independence, but mutual preferential independence does not imply additive independence. Imposing additive independence means that the individual is indifferent between the treatment scenarios: $T_{1}=\left\langle\left(q_{t}^{0}, q_{t+1}^{0}\right), \frac{1}{2},\left(q_{t}^{*}, q_{t+1}^{*}\right)\right\rangle$ and $T_{2}=\left\langle\left(q_{t}^{*}, q_{t+1}^{0}\right), \frac{1}{2}\right.$, $\left.\left(q_{t}^{0}, q_{t+1}^{*}\right)\right\rangle$, for any two periods $t$ and $t+1$. This is true, given that the marginal probabilities, which are the only basis for decision making in case additive independence holds, are equal and thus there should be indifference between the treatment scenarios.

The final two conditions that have to be imposed are, as in the case of preferences under certainty, stable preferences over lifetime and equal scaling constants. Stable preferences imply that the utility function for health can be written as

$$
u(q)=\sum_{t=1}^{T} k_{t} u\left(q_{t}\right)
$$

The assumption of equal scaling constants implies that

$$
\frac{1}{c} u(q)=\sum_{t=1}^{T} u\left(q_{t}\right)
$$


where $\left(1 / k_{t}\right) u(q)$ measures the number of QALYs and $c=k_{1}=k_{2}=\ldots=k_{T}$. Note that $\left(1 / k_{t}\right) u(q)$ is still a utility function, since the utility function is unique up to a positive linear transformation and $0<c<1$. As in the certainty case, equal scaling constants imply that improvements in health status are equally important across periods.

Since HYEs impose no additional ${ }^{7}$ restrictions on the individual's utility function for health, the following assumptions are sufficient for QALYs and HYEs to yield identical results: (1) additive independence of health status levels in the various periods; (2) stable intertemporal preferences for health status; (3) equal weights attached to health improvements in various periods. Both the QALY-approach and the HYE-approach assume that the single-attribute utility function for health exists. That is, health and non-health attributes in the individual's overall intertemporal utility function should be mutually utility independent.

\section{Recent challenges of the HYE model}

Recently the HYE approach has been challenged by various authors (Buckingham, 1993; Culyer and Wagstaff, 1993; Johannesson et al., 1993). Taken together, these authors raise three important points of criticism against the HYE: (1) The two-stage procedure to measure HYEs is nothing more than a complicated way of asking the time trade-off (Buckingham; Culyer and Wagstaff; Johannesson et al.)

(2) HYEs are identical to QALY scores obtained from a time trade-off experiment (Culyer and Wagstaff).

(3) HYEs assume risk neutrality with respect to healthy years (Johannesson et al.).

Since I have argued in Sections 4 and 5 that QALYs and HYEs are only equivalent under certain restrictive assumptions, the above papers clearly challenge the results derived thus far.

The first point mentioned above, i.e. the assertion that measuring HYEs by the two-stage standard gamble method proposed in Mehrez and Gafni (1991) will give identical results as measuring HYEs by a TTO question, is strictly speaking not the topic of this paper, since the point is directed at the measurement procedure of the HYE rather than at the concept itself. Gafni et al. (1993) have responded to this objection by pointing out that measuring HYEs by the TTO method establishes equality between $v\left(Q_{H}\right)$ and $v\left(Q_{T}\right)$ whereas measuring HYEs by the two-stage procedure establishes equality between $u\left(Q_{H^{*}}\right)$ and $u\left(Q_{T}\right)$. It should be clear from Sections 4 and 5 that in general value functions and utility functions are not identical and that there is no straightforward relationship between the two. Dyer and Sarin (1979, Theorems 4 and 5) have proved that a (measurable) value

\footnotetext{
${ }^{7}$ Again it has to be assumed that the utility function is increasing in healthy years.
} 
function and a vNM utility function are only equivalent in the special case where mutual preferential independence and additive independence of the attributes are satisfied. However, from this argument it does not follow that HYEs elicited by the two stage procedure will differ from HYEs elicited by the TTO procedure. Value functions and utility functions differ in that they (generally) assign a different number to the same indifference class of consequences. This does not imply though that the consequences contained in an indifference class will be different. Indeed, as Loomes (1995) shows, under transitivity and monotonicity with respect to healthy years the number of HYEs elicited by the two stage procedure will be equal to the number of HYEs elicited by the TTO method.

From the equivalence of the two measurement methods it does not follow that the second point of criticism, raised by Culyer and Wagstaff, that HYEs are as restrictive as QALY scores obtained from a time trade-off experiment, is correct though. Both the TTO-based QALY and the HYE start by reducing health scenarios to their equivalent number of years in perfect health by eliciting the value of $H^{*}$ for which $v\left(Q_{H^{*}}\right)=v\left(Q_{T}\right)$. This determines the number of HYEs and as long as monotonicity with respect to healthy years holds, one can use this number to consistently rank health scenarios according to the individual's preferences. The TTO-based QALY approach makes two additional assumptions with respect to the individual's preference structure. First it is assumed that the profile $Q_{T}$ can be reduced to a profile of constant health status $\left(q^{1}\right)$, which is a continuity assumption with respect to health status, and second that the value $H^{*} / T$ can be attached to $q^{1}$ and can be used in subsequent analyses where the number of years in $q^{1}$ is not necessarily equal to $T$. This is only justified if the assumptions outlined in Section 4 hold.

Finally, the claim made by Johannesson et al., that HYEs assume risk neutrality with respect to healthy life years, is based on the misconception that a HYE is a utility, that can be used in expected utility calculations, rather than an argument in the utility function. If the utility function for health is increasing in healthy years, HYEs will be a correct representation of the individual preferences for health without having to impose any further restriction on this utility function.

\section{An assessment of the various assumptions}

Having argued that HYEs and QALYs can theoretically differ, it can be asked whether it is likely that in practice they will differ. This amounts to an empirical assessment of the various assumptions. The present section will show that the QALY assumptions have been violated. It should be borne in mind though that the empirical evidence relates to the basic QALY model. This raises the question about what is intrinsic to a QALY. In my view, given that a QALY is essentially a weighting scheme in which life years are adjusted for quality of life levels, separability of life years and quality of life is essential to the QALY. This makes 
the independence assumptions essential for a QALY-type of health outcome measure. The assumptions of stable preferences and equal weights to health improvements are merely "convenience assumptions" which could be relaxed. Note though that these "convenience assumptions" are typically made in empirical work, given that in their absence the assessment task becomes highly involved.

\subsection{The certainty case}

It has been shown in Section 4 that under certainty, the QALY model relies on three assumptions: mutual prefential independence, stable intertemporal preferences and equal weights being attached to health improvements.

Mutual preferential independence over periods implies that preferences for health status in any two subsequent periods do not depend on the levels of health status in the other periods. Note first that this assumption is in clear conflict with one of the major contributions to the theory of health economics: the model of the demand for health by Michael Grossman (1972). Moreover, intuitively one would not expect mutual preferential independence to hold for every health profile. Especially in case preferences between the pairs $\left(q_{t}^{1}, q_{t+1}^{1}\right)$ and $\left(q_{t}^{0}, q_{t+1}^{0}\right)$ are being considered with $q^{1}$ denoting a health status level only slightly preferred to death, one would expect these preferences to be influenced by health status levels in other periods. Evidence of this has been reported by Sutherland et al. (1982). Sutherland et al. showed that attitudes toward survival in various health states change with the time of additional increments in survival in these health states. A majority of their subjects preferred three months of survival (followed by death) to immediate death, even in highly dysfunctional health states. However, for the most dysfunctional health states a majority of subjects preferred immediate death to 8 years of survival in these health states (followed by death). These findings suggest the existence of some sort of threshold ("maximal endurable time"' as Sutherland et al. call it) above which increments in survival are negatively valued. Note that the existence of a "maximal endurable time" is in conflict with mutual preferential independence: preferences for health states within a period cannot be considered without taking into account health status levels in other periods. It also suggests that mutual preferential independence is most likely to hold either when the deviation from normal health takes place for a relatively short period or when the deviation is not severe. Unfortunately it is precisely in the evaluation of severe and/or chronic conditions that the QALY approach has typically been applied.

It is easy to show that equal scaling constants $\tau_{i}$ and stable preferences over time together imply a constant proportional trade-off of life years for health status. The available empirical evidence does not support this constant proportional trade-off assumption. Sackett and Torrance (1978), measuring the values of different health states by the time trade-off technique, found evidence of an increasing rather than a constant proportional trade-off: the value both patients and members from the general public assigned to various health states decreased 
dramatically with increases in the number of years spent in those health states. Pliskin et al. (1980) also confronted the subjects in their study with some time trade-off questions. Out of 30 questions only 9 answers were consistent with a constant proportional trade-off. Of these 9 consistent answers, 5 were such that the subject indicated that he/she was willing to trade-off no life years at all against improvements in health status. Eliminating these cases leaves only 4 out of 25 cases that were consistent with the constant proportional trade-off assumption.

If the constant proportional trade-off assumption is not tenable, it can be asked which of the two constituent assumptions, stable intertemporal preferences or equal weights being given to health improvements across periods, is most likely to cause the violation. Positive time preference has the effect of imposing different values for the $\tau_{i}$ 's. More precisely, less weight will be given to health status levels further away in time and this can explain why increasing proportional trade-offs have been observed. The common practice of discounting QALYs can capture this phenomenon. However, discounting is unlikely to solve all problems. As empirical evidence suggests (Loomes and McKenzie, 1989), people attach different weights to being healthy at various stages of the life-cycle. Ideally the scaling factors should reflect this by giving more weight to the value function for health status in those years (generally childhood and early parenthood). However, simply discounting QALYs will not adequately represent this.

Concerning the stability of preferences over time, Sackett and Torrance (1978) found that elderly have somewhat different preferences for health status as assessed by the time trade-off method than younger people. The differences are small though. Moreover, even if significant differences would have been obtained, this would only have counted as weak evidence. Strong evidence can only be obtained by cohort studies. To date no such studies have been reported.

\subsection{The uncertainty case}

In Section 5 it has been shown that under uncertainty the QALY model imposes additive independence, stable intertemporal preferences and equal weights for equal health improvements across periods on the individual's utility function.

Additive independence is a strong condition. It implies indifference between level health status streams and single-period health status. That is, the life-time certainty equivalent $\boldsymbol{q}^{+}=\left(q_{1}^{+}, \ldots, q_{T}^{+}\right)$for the treatment scenario $\left\langle\boldsymbol{q}^{*}, \frac{1}{2}, \boldsymbol{q}^{0}\right\rangle$ where the $q$-vectors indicate life-time health status level streams, and the one-period certainty equivalent $q_{t}^{++}$for the treatment scenario $\left\langle q_{t}^{*}, \frac{1}{2}, q_{t}^{0}\right\rangle$, are by additive independence equal. Also as has been noticed before, additive independence implies indifference between the treatment scenarios $T_{1}=\left\langle\left(q_{t}^{*}, q_{t+1}^{*}\right), \frac{1}{2}\right.$, $\left.\left(q_{t}^{0}, q_{t+1}^{0}\right)\right\rangle$ and $T_{2}=\left\langle\left(q_{t}^{*}, q_{t+1}^{0}\right), \frac{1}{2},\left(q_{t}^{0}, q_{t+1}^{*}\right)\right\rangle$.

Intuitively, one would not expect additive independence to hold. People are generally more risk averse with respect to life-time streams than with respect to 
one-period streams and, therefore, it can be expected that $q_{t}^{+}<q_{t}^{++}$. Moreover, mixed scenarios such as $T_{2}$ will generally be preferred to extreme scenarios such as $T_{1}$.

No direct empirical evidence exists on the appropriateness of the additive independence assumption. Torrance et al. $(1982,1992)$ found clear evidence that the additive multi-attribute utility function was not appropriate, that is additive independence had to be rejected, in their evaluations of neonatal intensive care of very low birth weight infants and of long term sequelae of childhood cancer. Similarly, Eriksen and Keller (1993) rejected the additive specification of the multiattribute utility function for the toxicity and efficacy of drugs. However, the multi-attribute functions assessed in these studies are one-period functions. It might be questioned though whether, given that additive independence does not hold for one-period multi-attribute utility functions, it is reasonable to assume that it will hold for multi-period multi-attribute utility functions.

If additive independence is considered to be too strong a condition, mutual utility independence might still hold. To my knowledge mutual utility independence has not been examined with respect to preferences for health. Studies that have assessed multi-attribute utility functions for health status like the ones by Torrance et al. and Eriksen and Keller have typically assumed mutual utility independence to hold, given that without this assumption the task would become very tedious. One could hypothesize that the results of Sutherland et al. generalize to the case where preferences for uncertain lifetime health scenarios rather than preferences for certain scenarios are considered. That is, if confronted with a choice between health status level $q_{t}^{1}$ for certain and treatment with probability $p$ of success, it can in general not be assumed that the outcome of this choice problem is independent of the health status levels in the other periods. In case the results of Sutherland et al. do indeed carry over to preferences over uncertain outcomes, mutual utility independence is most likely to be violated in the case of relatively serious and/or chronic illnesses.

Together additive independence, equal scaling constants and stable preferences imply a constant proportional trade-off of life years for health status. Some additional empirical evidence is available about the appropriateness of this assumption under uncertainty. McNeil et al. (1981) found that individuals were only willing to trade-off life-years against improvements in health status level (in their study a change from less than perfect speech to normal speech) if the number of remaining life-years was more than five. Even if the number of remaining life-years was larger than 5 , individuals did not behave according to the constant proportional trade-off assumption, but rather according to the principle of increasing proportional trade-off. In principle both unequal scaling constants and unstable intertemporal preferences could cause this violation. More research on whether individuals place different weights on different phases in their life-cycle and on whether standard gamble valuations of health states vary with age is necessary before anything definitive can be said. 
The three QALY assumptions also impose risk neutrality with respect to life years. This is easily seen by observing that Eq. (12) is linear with respect to life years. McNeil et al. (1978) found risk aversion with respect to life years in a group of patients with operable bronchogenic carcinoma. The same authors also found risk aversion with respect to life years in a group of subjects from the general public in their study about the trade-offs between speech and survival (McNeil et al., 1981).

As the available evidence suggests, it should not be expected that the assumptions underlying the QALY model will in general be satisfied. Even though some of the basic QALY assumptions can in principle be relaxed, QALYs and HYEs are likely to yield different results. This will not necessarily lead to reversals of preferences though. It remains to be shown how likely these reversals are to occur in practice.

Finally, as has been outlined in Sections 4 and 5, both the QALY and the HYE approach assume that preferences for health can be considered separately, that is health and non-health attributes in the individual's preference structure are independent. Empirical evidence on the appropriateness of this assumption is scarce. Viscusi and Evans (1990) have studied wage-risk trade-offs in a sample of chemical workers. They found that the utility of wealth depended on the state of health. More precisely, the marginal utility of wealth decreased with decreases in health status level. This result challenges the assumption of an overall utility function in which all attributes are utility independent of their respective complements. Rejecting this assumption means rejecting the multilinear form of the utility function. Rejecting the multilinear specification of the utility function means rejecting the multiplicative and additive specifications since these are special cases of the multilinear utility function. More research is clearly necessary, but Viscusi and Evans' results suggest that even decision making based on HYEs may give misleading results.

\section{Possible pitfalls of HYEs}

Though, as has been argued above, a HYE will not by definition correctly represent the individual's preference structure, the HYE approach is theoretically sounder than the QALY approach. In using HYEs, one does not need to worry about the assumptions of additive independence, equal weights for equal improvements in health status in any phase of the life cycle and stable preferences over time. HYEs are more general than QALYs and therefore more likely to reflect individual preferences.

Unfortunately this greater theoretical soundness is achieved at a cost: eliciting HYEs is a rather time-consuming and complicated task. In case of a health care program yielding different possible health status outcomes and a probability 
distribution of survival years, the HYE task becomes very cumbersome. Mehrez and Gafni (1991) propose an approximation technique to solve this problem. However, if an approximation technique is used it can no longer be claimed that "the advantage of the HYE measure ... is that it stems directly from the individual utility function and thus fully reflects the individual's preferences', (Mehrez and Gafni, 1991). The price paid for an increase in practical feasibility is a decrease in theoretical soundness.

A further problem is associated with the use of HYEs as a societal decision rule. The HYE has been developed as an individual measure of preference. However, resource allocation decisions in health care are typically societal decisions, requiring the aggregation of the preferences of individuals. The HYE in its original form cannot address this kind of question since it gives no guidance concerning the aggregation procedure. Gafni and Birch (1991) have shown how HYEs can be made consistent with several aggregation procedures (equity algorithms as they call them). However, it should be noted that each of these equity algorithms imposes a specific type of utility function on the individuals constituting society. For example, in subscribing to the equity principle that a life in full health should be given equal weight for every member of society, society implicitly imposes the following type of utility function for health on its individuals:

$$
U(Q, T)=U\left(Q, T^{*}\right)+U\left(Q, T-T^{*}\right),
$$

where $T$ denotes the individual's total lifetime, $T^{*}$ denotes the individual's remaining lifetime and $T-T^{*}$ denotes the time lived so far by the individual.

It is somewhat contradictory that an approach which claims its superiority on the basis of imposing no restrictions whatsoever on the individual utility function for health, ends up by imposing restrictions on this utility function in order to be applicable in societal decision making concerning the allocation of health care resources. However, even as a societal decision rule, the HYE approach still allows more freedom to the individual utility function than the QALY approach.

Note that within the above adjustment algorithm there is an additional problem: the selection of an appropriate value for full lifetime $T$. As Gafni and Birch observe, either individuals who are not newly borns have to evaluate health states for negative periods of time or individuals have to perceive extreme lengths of life. It is a well-known result from the literature on choice under uncertainty that reference levels exert a major influence on individual risk attitudes (Schoemaker, 1982; Tversky and Kahneman, 1991; Luce et al., 1993). Setting unrealistic reference states is likely to lead to unrepresentative results. Using QALYs does not offer an easy way out of this problem. As Gafni and Birch have shown, applying QALYs as a societal decision rule requires making equity judgements as well. If one is willing to accept the equity principle that a life in full health should count equally for every individual, this requires, as in the HYE model, the specification of a reference state. 


\section{A compromise between QALYs and HYEs}

In the previous sections it has been argued that both QALYs and HYEs have their pros and cons. QALYs are only under fairly restrictive assumptions equal to utilities, but are easy to measure. HYEs on the other hand more closely reflect the individual's preference structure, though without being an exact representation of it in every situation. This greater theoretical soundness is achieved at a price: a more involved measurement procedure. Moreover, applying the HYE model as a societal decision rule involves sacrificing some theoretical soundness. It would be appealing to have a measure that combines the advantages of both measures, while at the same time avoiding their disadvantages. In this section an index is proposed that to some extent attains this rather ambitious goal.

The most restrictive assumption of the QALY-procedure seems to be additive independence of the health states in different periods. If additive independence is replaced by the weaker condition of mutual utility independence, the utility function for health becomes: ${ }^{8}$

$$
u(q)=1 / k\left\{\prod_{t=1}^{T}\left[1+k k_{t} u_{t}\left(q_{t}\right)\right]-1\right\},
$$

where

(1) $u($.$) and u_{t}\left(q_{t}\right)$ have been normalized,

(2) $k_{t}=u\left(q_{t}^{*}, q_{t}^{c 0}\right)$ with $q_{t}^{c}$ denoting the complement of $q_{t}$ and $\sum_{t} k_{t} \neq 1$,

(3) $k$ is a scaling constant that is a solution to

$$
1+k=\prod_{t=1}^{T}\left(1+k k_{t}\right)
$$

Equivalently, (20) can be written as

$$
k u(q)+1=\prod_{t=1}^{T}\left[1+k k_{t} u_{t}\left(q_{t}\right)\right]
$$

Recall that utility functions are unique up to a positive linear transformation. Thus, if $k>0$ then $u^{+}(q)=k u(q)+1$ and $u_{t}^{+}\left(q_{t}\right)=1+k k_{t} u_{t}\left(q_{t}\right)$ are also utility functions and

$$
u^{+}(q)=\prod_{t=1}^{T} u_{t}^{+}\left(q_{t}\right)
$$

\footnotetext{
${ }^{8}$ For a proof of this result see Keeney and Raiffa (1976).
} 
If $k<0$ then $u^{+}(q)=-[k u(q)+1]$ and $u_{t}^{+}\left(q_{t}\right)=-\left[1+k k_{t} u_{t}\left(q_{t}\right)\right]$ are utility functions so again

$$
u^{+}(q)=\prod_{t=1}^{T} u_{t}^{+}\left(q_{t}\right)
$$

So even when additive independence is relaxed to mutual utility independence, the procedure to calculate the number of utilities associated with a health care program is still rather simple.

One problem remains: the assessment of the scaling constants ( $k$ and the various $k_{\imath}$ 's). This task can be greatly simplified though by imposing the (convenience) assumption of equal $k_{t}$ 's for every phase in the life cycle. Under this assumption, the easiest way to proceed is by determining $k_{1}$ from the following standard gamble question: determine $p^{*}$ such that $\left(q_{1}^{*}, q_{2}^{0}, \ldots, q_{T}^{0}\right) \sim$ $\left\langle\left(q_{1}^{*}, \ldots, q_{T}^{*}\right), p^{*},\left(q_{1}^{0}, \ldots, q_{T}^{0}\right)\right\rangle$. That is compare the certainty of one year in full health with a treatment option which offers a probability $p^{*}$ of success (full health for the rest of life) and a probability $1-p^{*}$ of failure (immediate death). The indifference value $p^{*}$ is equal to $k_{1}$ which is equal to all other $k_{t}$ 's by assumption. Once the $T$ values of $k_{t}$ are known, $k$ can be solved from Eq. (21). This gives all the information necessary to calculate $u^{+}$from (24).

The proposed aggregation procedure depends on the adopted equity principle (Gafni and Birch, 1991). For example, if it is accepted that one healthy year should count equally for each individual, the procedure is as follows. Set the difference between $u\left(q_{t}^{*}\right)$ and $u\left(q_{t}^{0}\right)$ equal to 1 for every individual in each time period and aggregate these individual values into societal values. Similarly aggregate the various individual $k_{t}$ 's in a set of societal $k_{t}$ 's. From these the societal value of $k$ can be calculated.

In the derivation of the above index, use has been made of two assumptions: mutual utility independence and equal values of $k_{t} \cdot{ }^{9}$ It cannot be expected that these assumptions will hold in every situation. The assumption of equal values for the different $k_{t}$ 's can be made less restrictive by introducing discount rates. However, as has been noted above, this will not solve all problems. Alternatively, the weights associated with different phases of the life-cycle could be directly assessed by asking more questions. For obvious reasons, this requires replacing death as the worst health state. However, even then subjects may find it hard to imagine profiles of the type $\left(q_{1}^{0}, q_{2}^{0}, \ldots, q_{t-1}^{0}, q_{t}^{*}, q_{t+1}^{0}, \ldots, q_{T}^{0}\right)$. Therefore, a reformulation of the model in a disutility format is to be recommended. Such a procedure is more likely to produce reliable answers.

Even some violations of mutual utility independence are allowed. Since health status is made up of several dimensions, e.g. mobility, pain, self-care, the

\footnotetext{
${ }^{9}$ Note also that it is still assumed that the axioms of expected utility theory hold.
} 
attributes of the lifetime utility function for health are vectors consisting of scores on these various dimensions. That is, the various component utility functions $u_{t}($. are themselves multiattribute utility functions nested within a higher level multiattribute utility function. Nesting multiattribute utility functions provides additional degrees of freedom, which permit trade-offs between two attributes to depend on other attributes. This allows for some violation of the mutual utility independence assumption.

Stable intertemporal preferences can but need not necessarily be assumed. Calculating utilities for various age groups and testing whether these are significantly different seems the appropriate procedure before stability of preferences over time can be assumed.

\section{Concluding remarks}

It is not claimed that the above index is the ideal one. Still some restrictive assumptions have been made even though the implications of these assumptions can be relaxed by discounting, reformulation of the model in a disutility format and by nesting the component one-attribute utility functions. The above index attempts to combine the advantages of using QALYs (easy to calculate) with those of HYEs (theoretically sound at the individual level). The above index is easier to calculate than HYEs though not as easy as QALYs given that extra questions have to be asked to determine the $k_{t}$ 's. The index more closely approaches the individual utility function than the QALY model, given that less restrictive asumptions have been imposed on the individual's preference structure.

Problems arise when health status levels worse than death have to be evaluated. In such cases mutual utility independence is violated. Though nesting may solve some problems, extreme care should be taken in applying the index to evaluate health status levels worse than death. On the other hand, in such cases the QALY procedure cannot be applied either and the existence of HYEs is not guaranteed as Mehrez and Gafni (1991) show. How best to handle health outcomes worse than death remains an important issue on the research agenda for outcome measurement in health. At the moment, the recommended procedure is to use several measures and to test extensively for the sensitivity of the results obtained.

The step from QALYs to HYEs implied relaxing several restrictions that had been imposed on the individual's preference structure. The above index is in fact a step backwards on the road between QALYs and HYEs, since a restriction (mutual utility independence) has been re-introduced. An alternative course would be to take the opposite direction and investigate the implications of relaxing even more assumptions. This would lead to a sort of Grossman formulation in which the individual's overall utility function can no longer be assumed to be separable. Exploration of this road is an interesting topic for future research. 


\section{Acknowledgements}

I am grateful to Steve Birch, Eddy van Doorslaer, David Feeny, Amiram Gafni, Graham Loomes, Frans Rutten, George Torrance, Adam Wagstaff, Peter Wakker, Qinan Wang and two anonymous referees for valuable comments and suggestions on an earlier version of this paper. My thanks also goes to Angélique Visser for help in the preparation of successive manuscripts. Any remaining errors are my own. This work was partly supported by a grant from Merck Sharpe and Dome.

\section{Appendix 1}

Theorem 2 can be proved by combining Theorems 5.1 and 6.4 in Keeney and Raiffa (1976), which are based on results derived by Fishburn $(1965,1970)$.

Consider first an individual who only lives two periods. Given additive independence this individual will be indifferent between the treatment scenarios $\left\langle\left(q_{1}, q_{2}\right), \frac{1}{2},\left(q_{1}^{0}, q_{2}^{0}\right)\right\rangle$ and $\left\langle\left(q_{1}, q_{2}^{0}\right), \frac{1}{2},\left(q_{1}^{0}, q_{2}\right)\right\rangle$ since they have the same marginal probability distribution on quality of life levels in the two periods.

Equating expected utilities and setting $u\left(q_{1}^{0}, q_{2}^{0}\right)=0$, which is allowed given freedom to scale the utility function gives $\frac{1}{2} u\left(q_{1}, q_{2}\right)=\frac{1}{2} u\left(q_{1}, q_{2}^{0}\right)+\frac{1}{2} u\left(q_{1}^{0}, q_{2}\right)$.

Defining $u\left(q_{1}, q_{2}^{0}\right)=k_{1} u_{1}\left(q_{1}\right)$ and $u\left(q_{1}^{0}, q_{2}\right)=k_{2} u_{2}\left(q_{2}\right)$ to allow for free scaling of the single period utility functions gives the additive form of the multi-attribute utility function.

The generalization to the $T$ period case is straightforward. Define $Y$ as $\left\{q_{2}, \ldots, q_{T}\right\}$. Then from the above $u\left(Q_{T}\right)=k_{1} u_{1}\left(q_{1}\right)+k_{y} u_{y}\left(q_{2}, \ldots, q_{T}\right)$.

Break down $u_{Y}$ by defining $Z$ as $\left\{q_{3}, \ldots, q_{T}\right\}$. Apply the above again to yield: $u_{y}\left(q_{2}, \ldots, q_{T}\right)=k_{2} u_{2}\left(q_{2}\right)+k_{z} u_{z}\left(q_{3}, \ldots, q_{T}\right)$.

Proceeding this way and substituting the obtained expressions in each other gives the additive multi-attribute utility function.

That the additive multi-attribute utility function implies additive independence can immediately be derived by calculating the expected utilities of the treatment scenarios.

\section{References}

Buckingham, K., 1993, A note on HYE, Journal of Health Economics 12, 301-309.

Culyer, A.J. and A. Wagstaff, 1993, QALYs versus HYEs, Journal of Health Economics 12, 311 -323. Debreu, G., 1954, Representation of a preference ordering by a numerical function, in: R.M. Thrall, C.H. Coombs and R.L. Davis, eds., Decision processes (Wiley, New York) 159-165.

Debreu, G., 1960, Topological methods in cardinal utility theory, in: K.J. Arrow, S. Karlin and P. Suppes, eds., Mathematical methods in the social sciences (Stanford University Press) 16-26.

Debreu, G., 1964, Continuity properties of Paretian utility, International Economic Review 5, 285-293. 
Dyer, J.S. and R.K. Sarin, 1979, Measurable multiattribute value functions, Operations Research 27, $810-822$.

Eriksen, S. and L.R. Keller, 1993, A multiattribute utility function approach to weighing the risks and benefits of pharmaceutical agents, Medical Decision Making 13, 118-125.

Fishburn, P.C., 1965, Independence in utility theory with whole product sets, Operations Research 13, $28-45$.

Fishburn, P.C., 1970, Utility theory for decision making (Wiley, New York).

Gafni, A. and S. Birch, 1991, Equity considerations in utility-based measures of health outcomes in economic appraisals: An adjustment algorithm, Journal of Health Economics 10, 329-342.

Gafni, A., S. Birch and A. Mehrez, 1993, Economics, health and health economics: HYEs versus QALYs, Journal of Health Economics 12, 325-339.

Grossman, M., 1972, The demand for health: A theoretical and empirical investigation (National Bureau of Economic Research, New York).

Johannesson, M., J.S. Pliskin and M.C. Weinstein, 1993, Are healthy years equivalents an improvement over quality adjusted life years? Medical Decision Making 13, 281-286.

Karni, E. and D. Schmeidler, 1991, Utility theory with uncertainty, in: W. Hildenbrand and H. Sonnenschein, eds., Handbook of mathematical economics, Vol. 4 (North-Holland, Amsterdam) 1763-1831.

Keeney, R. and H. Raiffa, 1976, Decisions with multiple objectives: Preferences and value tradeoffs (Wiley, New York).

Koopmans, T.C., 1972, Representation of preference orderings over time, in: C.B. McGuire and R.Radner, eds., Decision and organization (North-Holland, Amsterdam) 79-100.

Krantz, D.H., R.D. Luce, P. Suppes and A. Tversky, 1971, Foundations of measurement, Vol. 1 (Academic Press, New York).

Loomes, G., 1995, The myth of the HYE, Journal of Health Economics.

Loomes, G. and L. McKenzie, 1989, The use of QALYs in health care decision making, Social Science and Medicine 28, 299-308.

Luce, R.D., B.A. Mellers and S-J Chang, 1993, Is choice the correct primitive? On using certainty equivalents and reference levels to predict choices among gambles, Journal of Risk and Uncertainty $6,115-143$.

McNeil, B.J., R. Weichselbaum and S.G. Pauker, 1978, Fallacy of the five-year survival in lung cancer, New England Journal of Medicine 299, 1397-1401.

McNeil, B.J., R. Weichselbaum and S.G. Pauker, 1981, Tradeoffs between quality and quantity of life in laryngeal cancer, New England Journal of Medicine 305, 982-987.

Mehrez, A. and A. Gafni, 1989, Quality-adjusted life years, utility theory, and healthy years equivalents, Medical Decision Making 9, 142-149.

Mehrez, A. and A. Gafni, 1991, The healthy years equivalents: How to measure them using the standard gamble approach, Medical Decision Making 11, 140-146.

Pliskin, J., D.S. Shepard and M.C. Weinstein, 1980, Utility functions for life years and health status, Operations Research 28, 206-224.

Sackett, D.L. and G.W. Torrance, 1978, The utility of different health states as perceived by the general public, Journal of Chronical Diseases 31, 697-704.

Schoemaker, P.J.H., 1982, The expected utility model: Its variants, purposes, evidence and limitations, Journal of Economic Literature 20, 529-563.

Sutherland, H.U., H. Llewellyn-Thomas, N.F. Boyd and J.E. Till, 1982, Attitudes toward quality of survival: The concept of "maximal endurable time", Medical Decision Making 2, 299-309.

Torrance, G.W., M.H. Boyle and P.H. Horwood, 1982, Application of multi-attribute utility theory to measure social preferences for health states, Operations Research 30, 1043-1069.

Torrance, G.W. and D. Feeny, 1989, Utilities and quality-adjusted life years, International Journal of Technology Assessment in Health Care 5, 559-575. 
Torrance, G.W., Y. Zhang, D. Feeny, W. Furlong and R. Barr, 1992, Multi-attribute preference functions for a comprehensive health status classification system, McMaster University CHEPA working paper no. 92-18.

Tversky, A. and D. Kahneman, 1991, Loss aversion in riskless choices: A reference dependent model, Quarterly Journal of Economics 106, 1039-1061.

Viscusi, W.K. and W.N. Evans, 1990, Utility functions that depend on health status: Estimates and economic implications, American Economic Review 80, 353-374.

Weinstein, M.C., H.C. Fineberg et al., 1980, Clinical decision analysis (W.B. Saunders, Philadelphia, PA). 